\title{
Petits aménagements hydroélectriques et libre circulation des poissons migrateurs
}

\author{
Small-scale hydropower schemes and migratory fish passage \\ par M. Larinier \\ CSP-CEMAGREF, GHAAPPE
}

et F. Travade

Électricité de France, Direction des Études et Recherches

After a brief reminder of the reasons why migrating fish need to move freely up and down rivers, this article describes current techniques in the area of upstream fish passage facilities, emphasising the value and limitations of each type : poll-type fishways, fish locks and lifts, bypass channels and preliminary weirs. The authors then define what is meant by efficiency in this context, before going on to describe fishway monitoring methods. The second part of the article reviews current knowledge on dowstream fish migration through hydropower stations, focusing on damage caused by fish passing through turbines or by spillways, and on the design of facilities (physical and behavioural barriers) used to prevent fish from entering water intakes. The study highlights surface bypasses which have been used successfully to guide migrating fish safely past existing water intakes. The authors conclude by emphasising that whatever the facilities used, small-scale hydropower stations virtually always have some impact on migratory fish populations, especially when a series of installations along their migratory route causes the impact to become cumulative.

\section{I $\square$ LES EXIGENCES EN MATIÈRE DE LIBRE CIRCULATION DU POISSON}

Les populations de poissons dépendent étroitement des caractéristiques de l'habitat aquatique. Cette dépendance est particulièrement marquée chez les poissons migrateurs qui exigent des milieux différents pour le déroulement des phases principales de leur cycle biologique : reproduction, production de juvéniles, grossissement et production de géniteurs. La possibilité de circuler d'un milieu à l'autre est nécessaire à la survie de l'espèce [1].

Les migrateurs amphibiotiques (ou " grands migrateurs ") doivent obligatoirement changer de milieu au cours de leur cycle qui se déroule pour partie en eau douce et pour partie en mer, avec des trajets entre zones de reproduction et zones de grossissement pouvant atteindre plusieurs milliers de kilomètres.

Chez le saumon, l'alose, la lamproie et l'esturgeon (migrateurs potamotoques) la reproduction a lieu en eau douce et la phase de grossissement en milieu marin. La migration continentale est une migration de reproduction. Ces migrateurs savent reconnaître leur réseau hydrographique natal et viennent s'y reproduire avec un taux d'erreur très faible. Ce phénomène de retour au foyer (" homing ") repose principalement sur l'identification olfactive du cours d'eau. Il a pour conséquence que chaque bassin hydrographique pos- sède un stock qui lui est propre et qui constitue une unité de gestion incontournable.

Chez l'anguille (migrateur thalassotoque), la reproduction a lieu en mer (Mer des Sargasses) et la migration continentale est au contraire une migration trophique de colonisation. On n'observe pas de ce fait d'isolement reproducteur des individus issus de tel ou tel bassin : le stock est commun à l'ensemble de la façade atlantique européenne.

Les espèces holobiotiques réalisent leur cycle biologique entièrement en eau douce. Toutes les espèces se déplacent dans le réseau hydrographique [2], mais cette activité migratoire est plus ou moins importante pour le bon déroulement de leur cycle biologique. Chez des espèces telles que le brochet, la truite fario, la truite de lac, les zones indispensables aux phases successives du cycle biologique sont bien individualisées et souvent séparées par des distances importantes : les besoins migratoires sont stricts pour le maintien d'une population en bon état. Chez d'autres espèces telles que l'ablette ou le gardon, ces besoins sont moins marqués mais il est nécessaire de maintenir une circulation d'individus entre les biefs pour éviter l'isolement reproducteur.

En France, la loi sur la pêche et la gestion des ressources piscicoles (30 juin 1984) établit un classement de rivières dans lesquelles doit être assurée la libre circulation d'un certain nombre d'espèces migratrices qui à ce jour sont le saumon atlantique, la truite de mer, les lamproies (marine et 
fluviatile), les aloses (grande alose et alose finte), l'anguille pour les migrateurs amphibiotiques, la truite de lac, la truite commune, l'ombre et le brochet pour les migrateurs holobiotiques.

\section{II $\square$ IMPACT DES PETITES CENTRALES SUR LA MIGRATION}

Quelle que soit son utilisation, un barrage constitue un obstacle à la migration du poisson et les petits aménagements hydroélectriques ne font pas exception. Dans le cas des grands migrateurs, comme le saumon, l'espèce pourra disparaitre du cours d'eau, voire du bassin, lorsque le barrage commande l'accès à toutes les frayères. Au cours des 150 dernières années on a assisté, en France, à une réduction constante de l'aire de distribution des espèces de poissons migrateurs, réduction accélérée avec le temps jusqu'à une période très récente et particulièrement marquée chez les espèces amphibiotiques comme le saumon, la truite de mer, I'alose, la lamproie ou l'anguille. Dans la majorité des cas la régression ou la disparition des espèces migratrices est liée à la multiplication des obstacles à la libre circulation souvent aggravée par une surexploitation par la pêche et la dégradation du milieu. Ces obstacles sont la cause de la disparition de stocks entiers (saumon du Rhin, de la Seine, de la Garonne et la Dordogne), ou du cantonnement de certaines espèces dans une partie très réduite du réseau hydrographique (saumon de la Loire, alose du bassin Garonne-Dordogne). Dans le cas du saumon, il a souvent suffi de la construction d'un seul obstacle, situé à l'aval de toutes les zones de reproduction, pour voir disparaître en quelques années toute une population.

Pour faire face à cette situation, des plans de restauration et de protection (plan saumon, plans grands migrateurs puis plus récemment contrats " retour aux sources ") ont été initiés par le ministère chargé de l'Environnement depuis une vingtaine d'années. La législation sur la pêche de 1984 fait d'autre part obligation aux propriétaires d'obstacles situés sur les cours d'eau classés à migrateurs d'assurer la libre circulation du poisson, à la montaison comme à la dévalaison. Ces deux démarches se sont traduites par l'aménagement ou l'amélioration de plus de 400 passes à poissons depuis une quinzaine d'années en France, contribuant de façon déterminante aux progrès dans la technique de conception.

Les problèmes liés à la migration de dévalaison n'ont été abordés que plus récemment : les expérimentations ont d'abord porté sur l'estimation des dommages lors du transit des poissons à travers les turbines puis sur la mise au point de dispositifs spécifiques destinés à limiter l'entraînement des migrateurs dans les prises d'eau des centrales hydroélectriques.

On abordera successivement ces différents aspects de la libre circulation, en insistant sur la notion d'efficacité des ouvrages de franchissement et sur les méthodes et les techniques permettant d'apprécier cette dernière.

\section{III $\square$ LA MIGRATION DE MONTAISON : LES PASSES À POISSONS}

Les passes à poissons sont traditionnellement classées en passes à bassins successifs, passes à ralentisseurs, écluses, ascenseurs, rivières artificielles de contournement et prébarrages.

\subsection{Les passes à bassins successifs}

Le type de passe le plus fréquemment utilisé est la passe à bassins successifs, consistant en une série de bassins reliant le pied de l'obstacle au bief amont. Les critères de dimensionnement sont basés sur les capacités de nage et le comportement des espèces migratrices et sur des considérations plus hydrauliques basées sur le retour d'expérience ou sur des études sur modèles réduits physiques $[1,3,4]$.

La dénivellation entre deux bassins successifs, fonction des espèces migratrices considérées, est comprise entre $0,15 \mathrm{~m}$ et $0,60 \mathrm{~m}$, et plus fréquemment entre $0,25 \mathrm{~m}$ et $0,35 \mathrm{~m}$. Le volume des bassins est déterminé à partir de la puissance volumique dissipée maximale qui caractérise le niveau de turbulence et d'aération admissibles par les poissons, valeur variant de 100 à 200 watts par $\mathrm{m}^{3}$ suivant les espèces concernées. La longueur des bassins peut varier de $1,2 \mathrm{~m}$ environ pour une petite passe à truite à faible débit à plus de $5 \mathrm{~m}$ pour une passe à saumons dimensionnée pour un débit supérieur à $1 \mathrm{~m} / \mathrm{s}$ [1].

Le type de passe à bassins le plus courant en France est la passe à échancrures latérales profondes et à orifices noyés pour les ouvrages de moyenne importance et la passe à fentes verticales pour les ouvrages à gros débit. Elles ont l'avantage majeur de fonctionner correctement sans organe de régulation en tolérant des variations notables des niveaux d'eau amont et aval [5].

Plus de 150 passes à bassins ont été construites au cours des quinze dernières années en France. Le débit dans ces ouvrages, fonction avant tout de l'importance du cours d'eau, peut varier de moins de $100 \mathrm{l} / \mathrm{s}$ pour des passes à truites situées sur de petits ruisseaux à plusieurs $\mathrm{m} / \mathrm{s}$ sur les grands cours d'eau à migrateurs. C'est avant tout le débit dans l'ouvrage et la chute à franchir qui déterminent le coût des ouvrages qui peut varier sur une micro-centrale de moins de $100000 \mathrm{~F}$ (petite passe à truite) à plus de 1 million de francs (passe multispécifique sur un grand cours d'eau). L'expérience montre que lorsqu'une passe à bassins est correctement dimensionnée, elle est peu sélective et peut être franchie par la majorité des espèces migratrices.

\subsection{Les passes à ralentisseurs}

La passe à ralentisseurs se présente sous la forme d'un canal à forte pente $(15 \%$ à $20 \%)$ dans lequel est disposée une succession de déflecteurs régulièrement espacés. Ces déflecteurs donnent naissance à des courants hélicoïdaux qui assurent une forte dissipation d'énergie au sein de l'écoulement et une réduction importante des vitesses $[1,6,7]$. Le poisson franchit une passe à ralentisseurs d'une seule traite et ne peut se reposer entre les ralentisseurs. C'est pourquoi, lorsque la dénivellation devient importante, le migrateur doit fournir un effort intense et il convient de prévoir des bassins de repos tous les 1,5 à 2 mètres de chute. Les passes à ralentisseurs demeurent relativement sélectives et ne sont utilisées que pour les espèces possédant des capacités suffisantes en termes de vitesse de nage et d'endurance (salmonidés, grands cyprinidés d'eau vive) [1].

Certains dispositifs comportant des ralentisseurs de fond en bois en forme de chevrons peuvent être utilisés à la fois comme glissière à canoë-kayak et comme passe, mais ils restent très sélectifs et limités aux salmonidés grands migrateurs.

Les passes à ralentisseurs sont particulièrement adaptées aux cours d'eau de petite ou moyenne importance sur les- 
quels le débit disponible pour la passe n'est que de quelques centaines de $1 / \mathrm{s}$. Elles s'intègrent particulièrement bien dans les seuils de hauteur modérée, en particulier les anciennes chaussées de moulins. Plus d'une centaine de passes de ce type ont été installées depuis une quinzaine d'années, principalement sur les cours d'eau à grands salmonidés migrateurs de Bretagne et de Normandie. Leur coût varie de $100000 \mathrm{~F}$ à $300000 \mathrm{~F}$.

\section{- 3.3 Ecluses et ascenseurs}

Le principe de fonctionnement d'une écluse à poisson est très proche de celui d'une écluse de navigation. On attire le migrateur dans un bassin et on l'écluse comme on écluserait un bateau [1,8]. Quelques écluses à poissons ont été construites en France dans les années 1960-1970. A part de rares exceptions, leur fonctionnement ne s'est jamais révélé satisfaisant, le plus souvent suite à une conception discutable : ouvrages sous-dimensionnés, fonctionnement des vannes simplifié, attraction insuffisante [1]. La principale limite de ce type de dispositif est qu'il semble difficile sinon impossible d'optimiser son cycle de fonctionnement lorsque plusieurs espèces aux comportements très différents sont concernées. L'efficacité est en effet très liée au comportement du poisson, variable suivant l'espèce, lequel doit demeurer dans la chambre aval pendant toute la phase d'attrait, suivre le niveau de l'eau lors de la phase de remplissage et sortir de l'écluse avant la vidange. A l'heure actuelle, l'installation d'écluses est exceptionnelle. On lui préfère l'ascenseur dont la technologie a progressé au cours de ces dernières années.

Dans les ascenseurs, le poisson est directement piégé dans une nasse. A intervalles réguliers, cette nasse, dont la partie inférieure est constituée d'une cuve destinée à remonter le poisson dans un volume d'eau suffisant, est déversée en amont. Un débit d'attrait, provenant du bief supérieur et destiné à attirer et à inciter le poisson à pénétrer dans la nasse, est injecté en amont de celle-ci après dissipation de son énergie $[2,3]$. Le coût d'un ascenseur, indépendant de la hauteur de chute, est fortement lié à l'importance du génie civil nécessaire à son intégration dans l'ouvrage : il varie de $300000 \mathrm{~F}$ à $2000000 \mathrm{~F}$. L'installation d'un ascenseur sur une petite installation hydroélectrique ne se justifie que pour des chutes importantes, supérieures à 7 à 8 mètres.

\subsection{Rivières artificielles de contournement}

La passe de type "rivière artificielle" consiste à relier biefs amont et aval par un chenal creusé dans l'une des rives, chenal dont le fond et les parois sont garnis de rugosités ou d'obstacles (épis, seuils...) imitant en quelque sorte un cours d'eau naturel. Il est à prévoir que le recours à ce type d'ouvrage, s'intégrant très bien dans le paysage, devienne beaucoup plus fréquent dans l'avenir. Cependant la pente d'un tel ouvrage ne peut dépasser quelques pour cent (de l'ordre de $5 \%$ lorsque le débit à transiter demeure limité à quelques centaines de $1 / \mathrm{s}$ ), ce qui rend son implantation souvent difficile $[1,9]$.

\section{- 3.5 Prébarrages}

Les prébarrages constituent souvent une solution efficace et peu coûteuse pour permettre les franchissement des obstacles de faible hauteur. Ils sont formés de plusieurs seuils ou murs créant à l'aval de l'obstacle des grands bassins qui fractionnent la chute à franchir. Ces prébarrages sont généra- lement situés à proximité de l'une des deux rives pour en faciliter l'entretien. Sur les petits cours d'eau ils peuvent être implantés sans inconvénient sur toute la largeur de l'obstacle. Les prébarrages conviennent avant tout aux obstacles situés sur les cours d'eau à salmonidés [1].

\section{- 3.6 Passes à anguilles}

L'anguille possède de faibles capacités de nage mais présente la particularité de pouvoir progresser par reptation sur des substrats humides. On utilise cette faculté dans les passes spécifiquement conçues pour l'anguille constituées de rampes garnies d'un matériau permettant la reptation (branchage, paille, brosse...) et alimentées par un faible débit destiné à irriguer le substrat. Un débit d'attrait est délivré à l'aval de la rampe. Ces dispositifs peuvent être installés à l'intérieur de passes à poissons classiques (passes à bassins ou à ralentisseurs) et profiter ainsi de l'attrait de leur débit d'alimentation.

\section{- 3.7 Implantation des passes à poissons}

Les migrateurs doivent trouver le plus rapidement possible l'entrée de la passe lorsqu'ils arrivent au pied de l'obstacle. Lorsque la plus grande partie du débit est turbiné, les migrateurs seront généralement attirés au pied des turbines. Il conviendra, dans la mesure du possible évidemment, d'installer l'entrée de la passe à côté de la centrale. Par contre, lorsque les ouvrages évacuateurs déversent, le migrateur pourra se présenter soit du côté de la centrale, soit du côté du barrage. C'est le cas en particulier lorsque l'aménagement présente une dérivation. Il devient difficile de trancher, à savoir s'il est préférable d'installer la passe au barrage ou à l'usine. Le poisson peut avoir un comportement très différent suivant les conditions hydrologiques, c'est-à-dire soit se présenter à la centrale et rester piégé au pied des turbines, soit faire des incursions au barrage. On est de ce fait amené à installer fréquemment deux dispositifs de franchissement.

Il faut avoir conscience que le débit réservé minimal défini dans la loi ( $10 \%$ du module inter-annuel), s'il est généralement suffisant pour assurer physiquement la circulation du poisson dans un bras court-circuité (c'est-à-dire en générant un tirant d'eau suffisant pour permettre la nage du poisson), n'empêche pas pour autant l'attraction et le piégeage du poisson au pied des turbines du fait du comportement de celui-ci l'incitant le plus souvent à rechercher le passage vers les zones à fort débit. Dans les cas où l'installation d'un dispositif de franchissement à l'usine n'est pas possible - en particulier en cas de dérivation de longueur importante comportant des ouvrages d'amenée partiellement ou totalement en charge - l'efficacité d'un ouvrage de franchissement au barrage peut être subordonnée à l'installation d'une barrière physique (seuil ou écran électrique) interdisant l'accès du poisson dans le canal de fuite et à l'adoption d'un débit réservé dans le bras court-circuité significativement plus important que le minimum défini par la loi.

Le comportement du poisson n'est pas le seul facteur à considérer dans le choix du type de dispositif de franchissement et de son implantation. Il convient également, en effet, de prendre en compte l'exposition de l'ouvrage aux crues, sa protection contre les corps dérivants et son accessibilité, de façon à en assurer la surveillance, le contrôle et l'entretien. 


\section{IV — CONTRÔLE ET EFFICACITÉ DES PASSES À POISSONS}

\subsection{Notion d'efficacité}

L'obligation de résultat en matière de libre circulation du poisson imposée par la législation débouche sur la notion d'efficacité des passes à poissons, souvent délicate à définir et encore plus à démontrer. L'efficacité s'exprime en termes à la fois de pourcentage de la population franchissant l'obstacle et de retard à la migration, c'est-à-dire en durée que met la population ou la fraction de la population à franchir l'obstacle.

Le niveau d'efficacité à atteindre sur un site donné doit être défini par rapport aux objectifs biologiques recherchés. Il est donc relatif aux espèces considérées, au nombre d'obstacles sur le cours d'eau et à la position de l'obstacle sur l'axe migratoire. Dans une passe à saumons ou truites de mer située à l'aval des zones de frayères, on cherchera à faire transiter la totalité de la population migrante. Si de plus ce cours d'eau est équipé d'un grand nombre d'obstacles, on cherchera à minimiser les retards induits par ces obstacles de façon à ce que les migrateurs arrivent à temps sur les zones de reproduction. $\mathrm{Si}$, par contre, la passe est située à l'amont du cours d'eau au milieu de la zone de frayères, on pourra être moins exigeant sur son efficacité. Enfin, pour une passe à cyprinidés dont l'intérêt biologique est avant tout d'éviter la sectorisation des populations dans les différents biefs, il n'est pas nécessaire de chercher à faire transiter la totalité de la population présente à l'aval de l'obstacle. La passe sera considérée comme efficace si un "certain nombre" d'individus (en proportion "raisonnable" par rapport à la taille des populations) y transite [1].

Le niveau d'efficacité à atteindre pour les passes à poissons dépend également du degré d'aménagement du bassin : sur un cours d'eau comptant deux obstacles à la migration, l'installation de passes à poissons ayant une efficacité de $70 \%$ assure le passage de $50 \%$ des migrateurs qui se présentent à l'aval. Sur un cours d'eau comptant 10 obstacles, cette même efficacité moyenne de $70 \%$ assurerait seulement le passage de $3 \%$ des migrateurs et il faudrait que tous les dispositifs de franchissement aient une efficacité de $93 \%$ pour arriver au même résultat de $50 \%$ en amont.

L'efficacité d'un dispositif de franchissement dépend de plusieurs facteurs :

- son implantation au niveau de l'obstacle,

- les conditions hydrauliques au voisinage de son entrée qui ne doit être masquée ni par des écoulements issus des turbines ou des ouvrages évacuateurs, ni par des zones de recirculation ou d'eaux mortes,

- son débit (à l'échelle des débits du cours d'eau, de l'ordre de plusieurs pour cent),

- son hydraulique interne (vitesses, turbulence et nature des écoulements),

- son maintien dans les conditions de fonctionnement optimal qui exige un entretien régulier.

L'efficacité potentielle des passes à poissons est variable selon les espèces. Pour les salmonidés, une efficacité de $90 \%$ à $100 \%$ est couramment obtenue, par contre, pour l'alose, une efficacité de $50 \%$ peut être considérée comme excellente et de $10 \%$ à $20 \%$ fréquente. Très peu de données sont disponibles sur les espèces autres que les salmonidés et les clupéidés.
L'expérience française et étrangère montre que les causes les plus fréquentes d'inefficacité des dispositifs de franchissement sont un manque d'attractivité (les poissons ayant du mal à trouver l'entrée de la passe, suite à une mauvaise implantation ou à un débit insuffisant) et un défaut d'entretien de l'ouvrage.

\subsection{Contrôle de l'efficacité}

Les opérations de contrôle des ouvrages de franchissement sont importantes pour la vérification de l'efficacité des ouvrages après leur mise en service ; elles permettent parallèlement d'accéder à une meilleure connaissance des populations de poissons migrateurs et des caractéristiques de leurs migrations.

Les trois techniques de comptage des poissons dans les passes les plus fréquemment utilisées au niveau des petites installations hydroélectriques sont le piégeage, le compteur à résistivité et le contrôle visuel $[1,10]$.

Le piégeage est facile à mettre en œuvre sur les petits ouvrages et offre l'avantage de permettre le recueil de données biométriques sur les migrateurs.

Le compteur à résistivité est constitué d'un capteur installé dans une passe qui détecte automatiquement le passage du poisson du fait de sa résistivité différente de celle de l'eau. Ce dispositif ne peut pas distinguer les espèces mais est capable de différencier deux à trois tailles de poissons. Il est donc de ce fait utilisé sur les cours d'eau salmonicoles où cohabitent un faible nombre d'espèces que l'on peut distinguer par la taille : saumon et truite fario par exemple.

Le comptage visuel, qui nécessite de faire passer les poissons devant une vitre verticale immergée, a l'avantage de permettre l'identification de la majorité des espèces sans manipulation. L'utilisation des techniques de vidéo-surveillance et d'analyse d'image permettent un comptage en continu avec une charge de personnel raisonnable.

On mentionnera également le radiopistage, qui consiste à suivre à distance des poissons porteurs d'émetteurs radio miniaturisés. C'est une technique précieuse pour évaluer globalement l'efficacité des dispositifs de franchissement et l'effet cumulatif des différents barrages sur un axe de migration. Une étude a été lancée il y a deux ans sur le Gave de Pau pour évaluer la franchissabilité d'une trentaine d'obstacles à la montaison. Elle a montré que les principaux retards à la migration pouvaient être attribués à un manque d'attractivité des dispositifs de franchissement et à un entretien notoirement insuffisant de ces dispositifs [11].

\section{LA MIGRATION DE DÉVALAISON}

\section{- 6.1 Dommages subis lors du passage dans les déversoirs et les turbines}

La construction d'une passe à poissons ne résout pas tous les problèmes de survie des espèces migratrices : outre le passage dans le sens aval-amont, il est nécessaire d'assurer la descente des juvéniles, voire des adultes pour certaines espèces. Lorsque, en période de dévalaison, la plus grande partie du débit est turbiné, les juvéniles sont entraînés dans les canaux d'amenée et, si l'espacement des grilles de prise d'eau le leur permet, passent à travers les turbines.

La technique des dispositifs destinés à assurer la dévalaison est beaucoup moins avancée que celle portant sur les passes à poissons : cela tient à ce que le problème de la dévalaison n'a été que plus récemment abordé, et surtout à 
ce que la mise au point de dispositifs efficaces est beaucoup plus délicate et complexe que celle des passes à poissons. Aucun pays ne peut prétendre avoir résolu de manière vraiment satisfaisante les problèmes liés à la dévalaison.

Le passage par les déversoirs et évacuateurs de crues peut entraîner des mortalités directes (blessures par cisaillement hydraulique, chocs sur les radiers ou dissipateurs d'énergie, variations brutales des vitesses et des pressions...) ou indirectes (sensibilité accrue à la prédation des poissons choqués ou désorientés) [12]. En France, sur les petites centrales, le passage des poissons par les ouvrages évacuateurs est rarement un problème : les hauteurs de chute des aménagements situés sur les cours d'eau à migrateurs sont généralement modérées, inférieures à une dizaine de mètres, les ouvrages évacuateurs constituant le plus souvent le meilleur chemin pour minimiser les dommages lors de la dévalaison, à condition que la réception des poissons à l'aval se fasse dans de bonnes conditions (présence d'une fosse d'une profondeur suffisante et absence de dissipateurs d'énergie).

Le passage à travers les turbines hydrauliques soumet le poisson à diverses contraintes susceptibles d'entraîner des mortalités importantes : risques de chocs contre les parties fixes ou mobiles de la turbine, accélération et décélération brutales, variations très brutales de pression. De nombreuses expérimentations ont été menées dans divers pays (EtatsUnis, Canada, Suède, Ecosse, Allemagne, France), principalement sur les juvéniles de salmonidés, et plus rarement sur les clupéidés (aloses) et les anguilles, pour déterminer les mortalités résultant du transit dans les principaux types de turbines $[13,14,15]$. Les résultats obtenus sont concordants, et permettent de tirer certaines conclusions générales sur les dommages potentiels subis par les poissons.

Les mortalités sont très importantes sinon totales dans les turbines à action (du type Pelton par exemple), mais fort heureusement ces turbines, réservées aux très hautes chutes, ne sont que très rarement implantées sur les cours d'eau à grands migrateurs. Elles peuvent cependant concerner les cours d'eau à truite.

Les mortalités des juvéniles de salmonidés dans les turbines Francis et Kaplan varient fortement en fonction des caractéristiques de la roue (diamètre, vitesse de rotation...), de leur régime de fonctionnement, de la hauteur de chute ainsi que de l'espèce et de la taille du poisson concerné : de moins de $5 \%$ à plus de $90 \%$ dans les turbines Francis, de l'ordre de $5 \%$ à $20 \%$ dans les turbines Kaplan. Généralement les microcentrales sont équipées de turbines de petite à moyenne taille qui sont susceptibles de tourner à des vitesses élevées, et peuvent de ce fait entraîner des mortalités importantes.

Les mortalités peuvent s'avérer plus importantes sur certaines espèces, plus sensibles aux variations de pression et surtout sur l'anguille, à cause de sa taille. Pour l'anguille adulte, on peut considérer une mortalité minimale de $15 \%$ à $20 \%$ dans les très grosses turbines de basse chute (contre quelques pour-cents pour les salmonidés) et $50 \%$ à $100 \%$ dans les turbines de plus petite taille équipant la plupart des micro-centrales installées sur les cours d'eau français.

\section{- 5.2 Les dispositifs de dévalaison}

Une première solution pour empêcher le transit des poissons par les turbines consiste à les arrêter au droit des prises d'eau par une barrière physique (grille de maille inférieure à leur taille) associée à un exutoire (by-pass) permettant au poisson de contourner l'usine et de rejoindre le bief aval.
L'installation de telles grilles requiert des vitesses d'écoulement à l'approche des prises d'eau suffisamment faibles pour permettre aux poissons de nager pendant le temps suffisant à la découverte de l'exutoire, faute de quoi les mortalités par " placage" sur les grilles peuvent devenir supérieures à celles subies lors du transit dans les turbines $[1,16]$.

Ces grilles doivent guider les poissons vers l'exutoire. De ce fait, la configuration la plus efficace est de disposer ces grilles inclinées par rapport à l'écoulement, le by-pass étant situé dans la partie aval de la grille. Ce guidage peut être assuré en installant le plan de filtration vertical, mais incliné (entre $15^{\circ}$ et $30^{\circ}$ ) par rapport à la direction générale de l'écoulement, ou incliné par rapport à la verticale (entre $20^{\circ}$ et $40^{\circ}$ ). Dans le cas des grilles à barreaux, et pour des salmonidés, on peut considérer qu'un espacement inférieur au $1 / 10$ de la longueur du poisson s'avère suffisant pour bloquer ce dernier.

Il paraissait peu réaliste d'envisager en France, sur les aménagements existants, l'installation systématique de grilles fines qui obligeraient à redimensionner la plupart des prises d'eau. On a orienté les recherches vers une technologie moins lourde : l'utilisation d'exutoires de surface, en mettant à profit la répulsion naturelle qu'éprouvent les poissons à franchir les grilles conventionnelles des prises d'eau même si l'espacement inter-barreaux leur permet le passage. Des expérimentations $[17,18]$ ont été entreprises depuis plusieurs années dans le but de quantifier l'efficacité de tels exutoires (par marquage-recapture et radiopistage) et d'en dégager les critères d'implantation et de dimensionnement.

Les résultats obtenus font apparaître des efficacités variant de moins de $10 \%$ à près de $80 \%$, qui dépendent étroitement de plusieurs facteurs :

- la répulsivité des grilles de prise d'eau : elle est fonction non seulement de l'espacement des barreaux par rapport à la taille du poisson mais également de l'existence d'une composante de la vitesse d'écoulement tangentielle à la grille qui assure un guidage du poisson. Pour les smolts de saumons et de truite de mer, le pouvoir répulsif des grilles a été noté lors des différentes expérimentations jusqu'à des espacements de l'ordre de $3 \mathrm{~cm}$ à $3,5 \mathrm{~cm}$. Cet effet répulsif semble ensuite rapidement diminuer lorsque le clair entre barreaux augmente, - la possibilité pour le poisson de stationner un laps de temps suffisant devant les grilles qui nécessite une vitesse maximale d'écoulement de l'ordre de $3 \mathrm{~L} / \mathrm{s}$ ( $\mathrm{L}$ étant la longueur du poisson), soit 0,45 à $0,50 \mathrm{~m} / \mathrm{s}$ pour les smolts de saumon atlantique,

- la position des exutoires qui doivent être situés le plus près possible des grilles et sur la rive où se trouve la zone de concentration des poissons, caractérisée en général par un contre-courant ou une recirculation,

- le débit dans l'exutoire doit être à l'échelle des débits turbinés ( $2 \%$ à $10 \%$ environ). Il doit être ajusté au cas par cas à chaque site en fonction des autres paramètres influant sur l'efficacité des by-pass (localisation du by-pass, hydrodynamique, caractéristiques de la grille de prise d'eau...) : plus les autres paramètres seront défavorables et plus il sera nécessaire d'augmenter le débit dans le by-pass,

- les conditions hydrauliques à l'entrée de l'exutoire se révèlent fondamentales pour l'efficacité de celui-ci : absence de turbulences et de courants ascendants, accélération graduelle de l'écoulement vers l'entrée de l'exutoire,

L'augmentation de l'attractivité de ces exutoires par la lumière, compte tenu du fait que la majeure partie de la dévalaison se déroule durant la nuit, offre d'intéressantes perspec- 
tives. Les résultats obtenus lors de plusieurs expérimentations in-situ montrent que les poissons sont attirés dans les zones éclairées par des lampes à vapeur de mercure $[17,18]$.

L'exutoire paraît être une solution acceptable pour résoudre le problème de la dévalaison au niveau d'une centrale hydroélectrique lorsqu'une efficacité de $60 \%$ à $80 \%$ est considérée comme biologiquement suffisante. Par contre, dans le cas d'aménagements sur lesquels les mortalités à travers les turbines sont très importantes ou dans le cas de micro-centrales en série sur un axe de migration, il conviendra de trouver des solutions plus efficaces. Cela peut passer par une barrière physique ou par un système de capture et de transport des juvéniles à l'aval.

Un grand nombre de barrières comportementales (écrans hydrodynamiques, électriques, sonores, lumineux, à bulles...) destinées à guider les poissons vers des exutoires ont été testées $[1,16]$. Des résultats ponctuels ont été obtenus mais n'ont en général pas débouché sur des applications en grandeur réelle en raison de leur forte sensibilité aux conditions de milieu (qualité de l'eau, configuration du site...). Seul l'écran hydrodynamique ("louver") a été utilisé avec succès sur plusieurs installations, notamment en Amérique du Nord. Dans l'état actuel des connaissances, les barrières comportementales ne peuvent au mieux qu'améliorer l'efficacité d'exutoires associés à des grilles de prises d'eau ou à des barrières physiques mais elles ne sont pas suffisamment efficaces pour être utilisées seules.

\section{3 Efficacité des dispositifs pour la dévalaison}

Nous disposons exclusivement à ce jour en France de résultats sur les dispositifs spécifiques aux salmonidés (saumon, truite de mer). Pour les exutoires de dévalaison associés aux grilles de prise d'eau, on peut obtenir, dans les cas les plus favorables, des efficacités de l'ordre de $60 \%$ à $80 \%$. Il est possible d'obtenir des efficacités voisines de $100 \%$ à l'aide de barrières physiques mais les contraintes d'installation et de fonctionnement peuvent s'avérer très lourdes. Les barrières comportementales sont d'une efficacité moindre et très variable d'un site à l'autre, ce qui nécessite de les utiliser uniquement en complément d'un autre dispositif.

Les méthodes permettant d'évaluer l'efficacité des dispositifs de dévalaison sont en général plus délicates et lourdes à mettre en œuvre que celles afférentes aux passes à poissons. Trois types de techniques sont utilisables : marquage-recapture, marquage par transpondeurs passifs' (PIT tags) et radiopistage. Pour tenir compte de la variabilité des conditions hydrauliques devant les prises d'eau pouvant considérablement affecter l'efficacité des dispositifs de dévalaison, les tests doivent être conduits sur une durée suffisante (un mois environ) et sur une nombre de poissons conséquent (un millier au moins).

\section{VII $\square$ CONCLUSIONS}

Diverses mesures compensatoires (débit réservé, passes migratoires, dispositifs pour assurer la dévalaison) peuvent permettre de limiter les impacts d'une micro-centrale sur les populations de poissons migrateurs... Le non-respect du débit réservé, le colmatage par les corps dérivants et le défaut d'entretien des dispositifs de franchissement sont

1 Marque électronique de petite taille implantable sur le poisson et détectable lors de son passage dans ou au-dessus d'un capteur. autant de causes supplémentaires de blocage et de retard dans les migrations. La technologie permettant de résoudre de façon convenable la dévalaison au niveau des prises d'eau est encore mal maîtrisée pour les salmonidés migrateurs et quasiment absente pour certaines autres espèces comme l'anguille, une efficacité élevée étant a priori beaucoup plus difficile à obtenir que sur les passes migratoires. Compte tenu de leur efficacité toujours partielle, notamment en ce qui concerne les dispositifs de dévalaison, on ne peut raisonnablement envisager l'installation que d'un nombre très limité de micro-centrales sur un cours d'eau sur lequel on cherche à protéger ou restaurer le stock d'une espèce migratrice.

\section{BIBLIOGRAPHIE}

[1] LARINIER M., TRAVADE F., PORCHER J.P., GOSSET C., 1994 Passes à poissons : expertise et conception des ouvrages de franchissement. Conseil Supérieur de la Pêche, Paris, $336 \mathrm{p}$.

[2 ]TRAVADE F., LARINIER M., BOYER-BERNARD S., DARTIGUELONGUE J., 1996. Performance on of four fish pass installations recently built on two rivers in southwest France. Colloque Fish Migration and Fish bypass-channels, Vienne Autriche - Septembre 1996 (à paraittre).

[3] BELL M.C., 1986. Fisheries Handbook of engineering requirements and biological criteria. Fish. Eng. Res. Prog., U.S. Army Corps of Engineers, Portland, Oregon, $290 \mathrm{p}$.

[4] CLAY C.H., 1995. Design of fishways and other fish facilities (second edition). Ed. Lewis Publishers, 248 p.

[5] LARINIER M, 1996. Upstream and downstream fish passage experience in France. International Conference on Fish Migration and Fish Bypass-Channels, Vienne Autriche, $16 \mathrm{p}$.

[6] RAJARATNAM N., KATOPODIS C., 1984. Hydraulic of Denil fishways, J. Hydr. Eng., 110(9), 1219-1233.

179 KATOPODIS C., 1990. Advancing in the art of engineering fishways for upstream migrants. Proc. Int. Symp. on Fishways 90, Gifu, Japan, 19. 28.

[8] AITKEN P.L., DICKERSON L.H., MENZIES W.J.M., 1966. Fish passes and screens at water power works. Proc. Inst. Civ. Eng., 35, 29-57.

[9] D.V.W.K.. 1996. Fisch Aufstiegsanlagen. Bemessung, Getaltung, Funktionskontrolle. Deutscher Verband für Wasserwirtschaft und Kulturbau, Merkblatt 232, Bonn, $110 \mathrm{p}$

[10] TRAVADE F., 1990. Monitoring techniques for fish passes recently used in France. Proc. Int. Symp, on Fishways 90, Gifu, Japan, 119-126.

[11] CHANSEAU M., CROZE O., LARINIER M., 1997. Suivi par radiopistage de la migration anadrome du saumon atlantique sur la partie amont du Gave de Pau. Rapport GHAAPPE 97,01, 45 p.

[12] RUGGLES C.P., MURRAY D.G., 1983. A review of fish response to spillways. Freshwater and Anadromous Div., Resource Branch Dept. of Fisheries and Oceans, Halifax, Nova Scotia, Can. Tech. Rep. of Fisheries and Aquatic Sci. $1172,30 \mathrm{p}$

[13] BELL M.C., 1981. Updated compendium of the success of passages of small fish through turbines. Fish. Eng. Res. Prog., U.S. Army Corps of Eng., North Pacific Div., Portland, Oregon, 294 p.

[14] MONTEN E., 1985. Fish and turbines. Fish injuries during passage through power station turbines. Vattenfall, Stockholm, 111 p.

[15] LARINIER M., DARTIGUELONGUE J., 1989. La circulation des poissons migrateurs : le transit à travers les turbines des installations hydroélectriques. Bull. Fr. Pisc., 312-313, 94 p.

[16] EPRI, 1994. Research update of fish protection technologies for water intakes. EPRI TR-104122, 208 p.

[17] LARINIER M., TRAVADE F. 1996. Smolt behavior and downstream fish bypass efficiency at small hydroelectric plants in France. Pages 891902 in Leclerc M. et al editors. Proceedings of the second IARH Symposium on Habitats Hydraulics, Ecohydraulics 2000, Québec city, Canada.

[18] LARINIER M., TRAVADE F. 1997. The development and evaluation of downstream bypasses for juvenile salmonids at small hydroelectric plants in France. Proc. American Fisheries Society, 127 th annual meeting Monterey, California, August 24-28 1997. 